\title{
Itinéraires Itinéraires
}

Littérature, textes, cultures

2020-3 | 2021

Le rap, une poésie de performances

\section{La performance du personnage musical du rappeur dans l'espace audiovisuel : les cas exemplaires d'Ice Cube, de Vince Staples et de Tyler, The Creator}

Performance of the Rapper's Musical Persona in the Audiovisual Space: The Exemplary Cases of Ice Cube, Vince Staples, and Tyler, The Creator

\section{Charlotte Gagné-Dumais}

\section{OpenEdition}

Journals

Édition électronique

URL : https://journals.openedition.org/itineraires/8919

DOI : $10.4000 /$ itineraires.8919

ISSN : 2427-920X

Éditeur

Pléiade

Référence électronique

Charlotte Gagné-Dumais, « La performance du personnage musical du rappeur dans l'espace

audiovisuel : les cas exemplaires d'Ice Cube, de Vince Staples et de Tyler, The Creator », Itinéraires [En ligne], 2020-3 | 2021, mis en ligne le 09 décembre 2021, consulté le 12 décembre 2021. URL : http:// journals.openedition.org/itineraires/8919; DOI : https://doi.org/10.4000/itineraires.8919

Ce document a été généré automatiquement le 12 décembre 2021.

\section{(c)}

Itinéraires est mis à disposition selon les termes de la licence Creative Commons Attribution - Pas d'Utilisation Commerciale - Pas de Modification 4.0 International. 


\title{
La performance du personnage musical du rappeur dans l'espace audiovisuel : les cas exemplaires d'Ice Cube, de Vince Staples et de Tyler, The Creator
}

\author{
Performance of the Rapper's Musical Persona in the Audiovisual Space: The \\ Exemplary Cases of Ice Cube, Vince Staples, and Tyler, The Creator
}

\section{Charlotte Gagné-Dumais}

Une des notions fondamentales de la culture rap et hip-hop est celle de l'authenticité. Dans le rap américain, on dit qu'un rappeur est authentique parce qu'il est real et qu'il represent (les siens, sa communauté). Ice Cube et son groupe N.W.A. (fondé en 1986 à Compton aux États-Unis) sont des précurseurs. Comme le précise le journaliste Jeff Chang dans son essentiel livre sur la culture hip-hop, Can't Stop Won't Stop (2005) :

Ils ont surpassé les progressistes du hip-hop, se positionnant entre le courant dominant et ces voix [inaudibles]. Ils affirmaient que personne d'autre qu'eux ne parlait au nom du frère du coin de la rue, et ce, de manière forte, provocante et sans ambages. Straight Outta Compton a donc également marqué le début de l'obsession du hip-hop pour "le vrai". Désormais, les rappeurs devaient représenter'. (Chang 2005 : 328)

2 Le gangsta rap est un style de rap né dans les années 1980 aux États-Unis et qui gagne en popularité dans les banlieues de Los Angeles. Les paroles sont souvent provocatrices et violentes. Pour le chercheur Gabriel Lessard, "[le gangsta rap] constitue une dénonciation des inégalités et une tentative d'accorder une place nouvelle au récit afroaméricain en réinscrivant leur identité dans une idéologie de lutte et de résistance " (2007 : 197). À l'âge d'or du gangsta rap, dans les années 1990, l'authenticité est à la base de la crédibilité et du respect qu'on accorde au rappeur. Il suffirait qu'il faillisse à cette valeur pour être détrôné aux yeux de la communauté hip-hop, mais également auprès 
de la critique et du public. Peu à peu, le succès du rappeur est déterminé par son argent, sa suprématie sur ses pairs, son agressivité et sa misogynie; le gangsta rap devient «[une] musique difficile à défendre ${ }^{2} »$ (Chang 2005 : 327). Malgré une influence incontestable sur le rap contemporain, le gangsta rap et ses caractéristiques s'essoufflent dans les années 2010 et font place à d'autres styles musicaux et esthétiques comme le mumble rap, le cloud rap, le trap, le emo rap, etc. L'héritage du gangsta rap y est dénaturé : les symboles qui y étaient attachés ne sont plus signes d'une émancipation sociale vers une situation économique plus stable mais la simple célébration du succès et de l'accumulation de biens matériels. La valeur de l'authenticité est remise en question : qu'est-ce que le rappeur représente désormais?

Comme l'indique l'ethnomusicologue Fernando Orejuela dans son ouvrage majeur Rap and Hip Hop Culture (2015), le rappeur est avant tout interprète d'un personnage :

Les rappeurs tels que 2Pac, Bushwick Bill et Ice Cube prétendent que, dans leurs chansons, ils sont tantôt observateurs de l'environnement, tantôt participants. Le rappeur dépeint des images difficiles de la rue comme s'il ou elle l'expérimentait directement. Considérons le fait que tous les rappeurs ont des noms de MC - des alter ego semblables aux personnages de lutteurs ou de superhéros de bande dessinée : [...] O'Shea Jackson n'est pas Ice Cube ${ }^{3}$. (Orejuela $2015: 142$ )

Ainsi, quand le public définit un rappeur comme étant authentique, c'est qu'il attribue de la crédibilité à son personnage et à ce que celui-ci représente. La notion de personnage de cet article se base sur la conceptualisation du personnage musical (musical persona) que fait le professeur spécialiste des arts de la performance Philip Auslander (2006). Selon lui, «l'identité personnelle peut être vue comme une chose que l'on performe. La performance de soi dans la vie quotidienne se défend tout autant que la performance d'un texte dans un théâtre ou dans une salle de concert. Bref, l'objet direct du verbe performer n'est pas nécessairement quelque chose - il peut aussi être quelqu'un, une identité plutôt qu'un texte" ». Il ajoute que " quand on voit un musicien performer, nous ne voyons pas seulement la "vraie personne" jouer; comme pour les acteurs, il existe une entité qui sert de médiateur entre les musiciens et l'acte de performance. [...] Ce que les musiciens performent avant tout, ce n'est pas la musique, mais leur propre identité de musicien, leur personnage musical ${ }^{5}$ ». Cela est d'autant plus vrai pour les rappeurs car l'espace audiovisuel leur permet de déployer leur performance : les vidéoclips, les annonces publicitaires et les films donnent au rappeur une multitude d'outils médiatiques pour faire valoir et approfondir son personnage musical. En ce sens, la performance de leur persona se rapproche davantage de celle qu'un acteur fait de son personnage. De plus, Auslander insiste sur le fait que le personnage musical est défini également par des interactions sociales et pas nécessairement par la représentation directe de la personnalité du musicien (2006: 103). Je crois que l'espace audiovisuel peut être le lieu de ces interactions sociales déterminantes.

4 Dans le rap contemporain, certains rappeurs proposent une nouvelle façon de s'identifier aux valeurs de représentation et d'authenticité, en proposant chacun une distance différente entre eux-mêmes et leur personnage musical, et c'est par leur utilisation de l'espace audiovisuel qu'ils réussissent à diminuer ou à agrandir ces limites. Pour observer ce changement, je propose d'abord d'identifier comment le gangsta rap s'est imposé comme la fondation des codes de la culture rap tels qu'on les conçoit aujourd'hui grâce à une analyse de la carrière du rappeur et acteur Ice Cube. Celui-ci possède le statut de vétéran du rap : il est de ceux qui ont concrétisé la notion 
d'authenticité comme fondement du personnage du rappeur. De ce fait, il se présente comme un modèle de la relation au personnage musical lors de l'âge d'or du gangsta rap. Par la suite, je commenterai l'évolution du personnage du rappeur à travers les figures plus actuelles de Vince Staples et de Tyler, The Creator. Vince Staples remet en question l'authenticité du mouvement du gangsta rap en proposant son personnage invisible. Quant à Tyler, The Creator, il détourne la notion de l'authenticité par l'incarnation de personnages multiples. Ces deux rappeurs s'éloignent non seulement du modèle d'Ice Cube, mais proposent également des approches innovantes quant à la relation au personnage musical. Ces trois cas représentent en somme différents pôles et différents contextes de relation entre le rappeur et son personnage musical et l'on peut, à travers ceux-ci, brosser un portrait plus global de la fonction du personnage musical au sein des particularités de la musique rap et de la notion d'authenticité.

\section{Ice Cube et le personnage classique}

5 L'analyse de la performance d'Ice Cube permet d'identifier comment le mouvement du gangsta rap engendre la consécration de l'authenticité comme valeur clé de la culture rap. Sa carrière d'acteur a été déterminante et judicieusement réfléchie afin d'assurer son influence dans le milieu du rap.

O'Shea Jackson est né en 1969 et a vécu dans le quartier de South Central Los Angeles. Bien qu'il soit témoin de violence et de pauvreté, Jackson grandit dans une famille de la classe moyenne qui le soutient dans ses études et ses explorations musicales (Leonard 2007 : 294-295). En 1986, il adopte officiellement le pseudonyme Ice Cube et forme avec les rappeurs Dr. Dre (Andre Romelle Young) et Eazy-E (Eric Lynn Wright) le groupe $\mathrm{N}^{* * * * *}$ Wit Attitudes (N.W.A.). En 1988, N.W.A. publie son deuxième album et plus grand succès : Straight Outta Compton (Ruthless Records).

7 Dès cette époque, Ice Cube est un personnage. Jackson n'a jamais été membre d'un gang de rue, il n'a jamais fait de prison. Il a grandi dans un milieu modeste mais confortable : la réalité qu'il dépeint dans ses textes est celle dont il est témoin, non celle qu'il est amené à vivre directement. Orejuela explique que bien qu'ils puissent donner l'impression de s'exprimer comme des gangsters, ni Ice Cube ni ses comparses de N.W.A. n'ont appartenu à un gang de rue de Los Angeles (2015:125). Il se définit comme un journaliste car il témoigne d'une réalité dans ses textes (Leonard 2007 : 293-294). Pourtant, dans ses raps, Ice Cube se place rarement au rang de témoin, il s'inclut dans la narration qu'il crée. Ce qui le démarque des autres membres de N.W.A., ce sont ses textes plus engagés, politiques et dénonciateurs. En se faisant personnage des situations sociales qu'il dénonce, Ice Cube gagne en sympathie et en crédibilité. Christopher J. Bell, dans son article « Hip Hop Fancy: Performative Black Masculinity, Feminism, and the Clear Text of Ice Cube's Death Certificate " (2016), considère qu'en écrivant leurs textes à partir d'un point de vue intérieur à cette communauté (la jeunesse afro-américaine), les membres de N.W.A. peuvent dénoncer des enjeux sérieux, tels que la brutalité policière. Bell donne en exemple la chanson « Fuck Tha Police» de N.W.A. (Straight Outta Compton, Ruthless Records, 1988) : «En commençant par le pronom personnel "je", Cube utilise une tactique bien différente de celle des couplets de Ren et de Eazy pour toucher son auditeur : "Fuck the police comin straight from the underground/A young $\mathrm{n}^{* * * *}$ got it bad "cause I'm Brown" ${ }^{\prime} »$ (2016:21). Il ajoute: «Le rappeur ne s'arrête pas là dans sa façon de faire comprendre à tous la 
colère que lui et ses pairs ressentent. À ce moment-là, l'auditeur adhère à la rhétorique de Cube et croit que le rappeur ne rappe pas seulement à propos de lui, mais rappe pour son public $^{7} »(22)$. C'est parce qu'il se présente comme faisant partie de cette communauté qu'Ice Cube gagne d'emblée en crédibilité: la performance de son personnage musical semble collée à sa personne, même si son gangstérisme est aussi fictif qu'engagé. Chang explique comment Ice Cube est influent: "Le son était si puissant qu'il a fallu le nommer ; c'est pourquoi on a baptisé "gangsta rap" la musique de N.W.A. d'après l'hymne indomptable d'Ice Cube ${ }^{8}$ " $\left.2005: 320\right)$. Le professeur David J. Leonard argumente que N.W.A., et surtout Ice Cube, s'inscrivent dans l'authenticité essentielle au hip-hop et au gangsta rap :

Le succès de N.W.A. avec Straight Outta Compton reflète la dialectique de leur stratégie marketing, une stratégie qui repose sur la véracité et l'authenticité de leur musique [...]. En d'autres termes, le pouvoir des paroles de Cube et bien sûr Cube lui-même avec sa mine renfrognée et ses jurons incessants reflètent leur authenticité, un critère accentué par N.W.A. et adopté par leurs auditeurs9. (Leonard 2007 : 300)

Le personnage d'Ice Cube est reconnu comme étant authentique parce que le rappeur aura été capable de représenter une réalité à laquelle le public pouvait s'identifier. Il s'inscrit dans une image du réel qui, bien que peu alimentée par sa biographie, se défend par l'engagement qu'il manifeste dans ses textes et dans son attitude. Ice Cube étoffe la construction de son personnage avec son premier contrat d'acteur.

En 1991, Ice Cube est déjà une star au sein de la communauté hip-hop, statut qui lui permet de décrocher son premier rôle au cinéma. Ice Cube accepte le rôle de Doughboy dans le film Boyz $N$ the Hood du jeune réalisateur John Singleton (Colombia Pictures). Parce que sa réputation le précède, le rôle est écrit spécialement pour lui (Lobalzo Wright 2016 : 57). Mais le personnage de Doughboy ne sait contenir celui d'Ice Cube : «Doughboy est l'incarnation de ce dont traite Ice Cube en rappant: le jeune homme noir pris dans des gangs de rues et des activités criminelles, la pauvreté et le désespoir d'être socialement marginalisé dans son quartier, le père absent, le manque de perspectives éducatives, et la propension à finir en prison ${ }^{10}$ » (Lobalzo Wright 2016 : 58). Si, dans ses chansons, Ice Cube tentait de représenter une réalité à la manière d'un journaliste de terrain, voici qu'il l'incarne pleinement. D'autre part, la figure d'Ice Cube prend place dans celle de Doughboy et dépasse le cadre du film :

La contribution d'Ice Cube à Boyz transcende son incarnation de Doughboy : il écrit et enregistre la bande originale; il légitime le message et le ton du film par son attachement à South Central ; et sa popularité et ses attaches à la communauté hiphop contribuent au buzz médiatique et au succès du film au box-office ${ }^{11}$. (Leonard $2007: 310)$

Le film est un succès parce qu'on peut y voir et y entendre le personnage d'Ice Cube. Le rappeur lui-même concède que Doughboy lui sert à manipuler son image (Leonard 2007 : 305). Après le succès de sa performance dans Boyz $N$ the Hood, Ice Cube s'essaie à la scénarisation avec le film Friday (réalisation de F. Gary Gray, New Line Cinema, 1995) tout en continuant sa carrière musicale. Bien que Friday soit une comédie et qu'Ice Cube délaisse son air habituellement sérieux, le personnage de Craig Jones s'inscrit dans la continuité de son travail d'interprétation et représente le même type de personnage (Lobalzo Wright 2016: 58). Par ailleurs, les liens entre Doughboy, Craig Jones et Ice Cube sont plus qu'inopinés, ils sont conceptuels :

Peut-être pour souligner un changement de l'image typique d'Ice Cube, le personnage de Craig porte les mêmes vêtements dans la première scène de Friday que le personnage de Doughboy portait dans la dernière scène de Boyz $N$ the Hood. 
[...] Ice Cube utilise Friday pour construire le récit de "It Was a Good Day", racontant le plaisir de passer du temps entre amis en dépit de la peine et de l'injustice vécues au quotidien ${ }^{12}$. (Leonard $2007: 311$ )

Le Ice Cube du clip «It Was a Good Day » est le Ice Cube de Friday: le personnage est omniprésent et traverse les différentes narrations auxquelles le rappeur participe. Suite à Friday, la carrière cinématographique d'Ice Cube ne fait que s'épanouir. Ice Cube fusionne la performance de son personnage musical à celles de ses personnages filmiques, floutant davantage les limites entre elles.

Aux yeux de ses fans, il importe peu qu'Ice Cube soit un personnage créé par O'Shea Jackson, tant qu'on lui accorde une véracité. Selon Auslander, l'implication des fans est en effet déterminante pour la construction du personnage musical: «Bien que l'on s'attende à ce que le personnage musical soit cohérent d'une performance à l'autre, il est également produit à chaque performance par un consensus établi avec le public. Le public est donc cocréateur du personnage musical et a un investissement qui va au-delà de la simple consommation ${ }^{13}{ }^{\prime}(2006: 117)$. Les fans croient en la crédibilité du personnage d'Ice Cube car elle est maintenue au fil de ses différentes manifestations audiovisuelles.

Alors que le personnage d'Ice Cube s'étoffe grâce au cinéma, la popularité de rappeurs plus contemporains grandit plutôt grâce à l'importance d'Internet et des réseaux sociaux : la production, puis le partage et la consommation rapides des vidéoclips sont un atout pour les rappeurs et l'établissement de leur relation avec leur public.

\section{Vince Staples et le personnage invisible}

11 Vince Staples est né en 1993 dans la ville de Long Beach, en Californie, où il connaît une jeunesse marquée par la pauvreté, le trafic de drogues, les gangs de rue et la brutalité policière. Son premier album, Summertime '06 (Def Jam) paraît en 2015. Le style de Vince Staples est critique, direct, parfois cynique ou ironique mais toujours juste. Sa carrière est marquée par le rejet d'une certaine esthétique devenue normalisée au sein de la culture hip-hop: Staples refuse les excès et le luxe. Contrairement à Ice Cube, qui véhicule aujourd'hui l'image de quelqu'un qui a dépassé son milieu social d'origine et qui est couronné de succès, Staples s'inscrit toujours au sein de sa communauté. Ses clips servent à souligner la dimension critique de ses textes, tout en imposant une esthétique minimaliste et réaliste : Staples se présente comme un anti-rappeur (Aguirre 2016). Tous les aspects de son travail visent à minimiser la distance entre lui-même et son personnage et par conséquent, à renforcer l'authenticité qu'on lui accorde. Staples est la figure du personnage invisible.

D'une part, Staples n'œuvre pas sous un pseudonyme : Vincent Staples est son vrai nom. D'autre part, il remet en question l'authenticité telle qu'elle était établie auparavant parce qu'il dénonce les faux-semblants de ses collègues et redirige l'attention du public vers des enjeux sociaux. Staples revendique la réelle authenticité promue par le gangsta rap dans les années 1990: issu des quartiers pauvres de Los Angeles et ayant connu la violence de ce milieu, Vince Staples ne fait pas semblant. La position qu'il prend dans ses textes est ancrée dans le réel et non à travers le point de vue fictionnel ou journalistique d'Ice Cube. Dans une entrevue, la poétesse Simone White confronte Staples à son personnage, suggérant que l'humain et le rappeur ne sont pas les mêmes personnes. Il répond: "Je fais de mon mieux pour qu'ils soient les 
mêmes. Il y a des choses que je ne partage pas. Je ne dévoile pas ma famille ou ma vie personnelle, mais pour ma musique, j'essaie d'être aussi ordinaire que possible. Je ne suis pas riche ni célèbre ni important, alors je ne prétends pas l'être. [...] C'est le problème avec les rappeurs, nous devons tous être riches ou célèbres ou des durs à cuire $^{14} »$ (White $2016: 156$ ). Cette approche est appuyée par son image : Staples est sans artifice.

Dès ses débuts, Vince Staples est ouvertement critique du gangsta rap. Dans son single « Norf Norf », Staples rappe : « One wrong word, start bustin'/Put that on my Yankee hat, I'm a gangsta Crip, fuck gangsta rap ${ }^{15} »$ (Summertime '06, Def Jam, 2013). Par ces lignes, il critique ce que l'image du gangsta rap est devenue. Staples dénonce l'authenticité brandie par certains artistes : ceux-ci prétendent être issus d'un milieu mais n'en proposent qu'un verni fait de codes esthétiques, de manifestations de pouvoir et de succès (quantité démesurée de biens matériels, imagerie violente, démonstrations de popularité). À l'inverse, l'esthétique de Staples est efficace et minimaliste. Les paroles de la chanson sont truffées de références critiques au hip-hop, mais font surtout le portrait de la vie difficile de Northside Long Beach: "Knowin' change gon' come like Obama and them say/But they shootin' everyday 'round my mama and them way" " ("Norf Norf», Summertime '06, Def Jam, 2013). Cette perspective est soulignée par les images du clip de la chanson, dont le traitement en noir et blanc assure une esthétique épurée. Les trente premières secondes sont un gros plan sur le visage de Staples. La caméra recule pour dévoiler que Staples est sur la banquette arrière d'une voiture de police alors que ses comparses se font arrêter. La nature du crime que Staples aurait commis ou non est sans importance : le clip agit comme commentaire sur le profilage racial de la police américaine (d'ailleurs, tous les prisonniers montrés dans le clip sont des hommes racisés). Contrairement aux signifiants usuels de la culture rap, Staples ne se présente pas ici sur un mode triomphant, riche et puissant; il se dépeint plutôt victime du système, de violence et d'intimidation. Même si le vidéoclip met en scène une fiction, l'approche de Staples et l'esthétique du clip sont ancrées dans le réel, diminuant l'écart possible entre Staples et son personnage musical.

La présence en ligne de Staples contribue à la déconstruction du personnage : dans ses entrevues, il offre constamment un regard critique sur la culture rap et sur son travail. Cela s'est particulièrement manifesté lors des évènements entourant la controverse de sa chanson «Norf Norf»: une mère de famille (désormais reconnue sur Internet comme la Norf Norf Mom) s'est offensée des paroles de la chanson dans une vidéo devenue virale sur Internet. La femme s'indignait que la chanson puisse avoir une mauvaise influence sur ses enfants. Lors d'une entrevue accordée au magazine The Independant au sujet de la réaction de la Norf Norf Mom, Staples commente : «Ce n'est pas correct d'attaquer quelqu'un pour ses principes, ses opinions ou sa religion. Je crois que c'est très immature ${ }^{17} »$; il ajoute :

Nous avons déjà beaucoup de problèmes dans les relations entre les Noirs et les Blancs dans ce pays, basés sur des incompréhensions. Selon moi, elle n'a pas l'air d'une raciste. Elle n'a pas l'air d'une mauvaise personne. Mais ce n'est pas très responsable de la part des gens de prendre ça et d'y chercher un commentaire sur les enjeux [de cette communauté] ${ }^{18}$. (Carissimo 2016)

La réaction de Staples surprend son public : la maturité avec laquelle il réagit contribue à le caractériser comme un rappeur authentique, sans faux-semblants. Il favorise l'échange et l'empathie. 
Nonobstant, cette situation est recyclée avec humour et cynisme dans le vidéoclip de «FUN! », chanson tirée de son EP FM! (Def Jam, 2018). Fidèle à sa démarche, Staples se place au même niveau que les autres personnes présentes dans ses clips: les membres de sa communauté sont des personnages centraux au même titre que lui. Le clip de «FUN! » reprend les codes visuels de l'option Street View du site de Google Map. Staples se retrouve au centre d'une série de scènes banales à première vue, qui brossent un portrait réaliste et poignant de la vie du quartier de Northside Long Beach où il $\mathrm{a}$ grandi. Le clip offre un commentaire critique sur la façon dont les quartiers pauvres des communautés afro-américaines sont perçus : de loin, sans réelle intervention de la part des observateurs, une consommation sécurisée qui ostracise une réalité sociale. Le clou du vidéoclip est le dévoilement d'un jeune garçon blanc qui observait le quartier à travers son ordinateur, se révélant comme le manipulateur du Google Map mis en scène par le clip. Le clip se termine sur la voix de la mère du garçon qui l'appelle, et celui-ci refermant rapidement l'écran de son ordinateur. On ne peut s'empêcher de faire le rapprochement entre la fin du clip et les commentaires de la Norf Norf Mom. En faisant référence à des événements réels de sa carrière, et en utilisant des images réalistes, Staples renforce son personnage invisible. Sa démarche et ses propos, comme ses images, sont crus et directs.

Dans ses clips, Staples s'inspire des codes du documentaire pour s'inclure dans une communauté et une réalité. Il n'est pas animé par une volonté d'être porte-parole d'une cause, mais adresse certains enjeux avec simplicité et sincérité. Staples refuse une position individualiste qui viendrait suggérer que son succès artistique (et monétaire) le place au-dessus de sa communauté, il entretient toujours un rapport inclusif avec celle-ci. Cette volonté et son sens esthétique font qu'on accorde à Staples une aura d'ultra-réalisme, brouillant plus que jamais les limites entre l'homme et son personnage. Parce qu'il en est toujours critique, l'authenticité de Staples dépasse celle portée par le gangsta rap. D'une tout autre manière, le rappeur Tyler, The Creator remet lui aussi en question les fondements de la culture rap. Ses choix esthétiques, radicalement opposés à ceux de Staples, font de lui un personnage excentrique et inclassable. Son image lui sert à explorer différents personnages et différents points de vue.

\section{Tyler, The Creator et le personnage multiple}

17 Tyler Okonma est né à Los Angeles en 1991. Il connaît ses premiers succès en tant que membre éminent du collectif Odd Future Wolf Gang Kill Them All (OFWGKTA) fondé en 2007. En 2009, il commence sa carrière solo avec l'album controversé Bastard, qu'il autoproduit.

18 Avant tout, il faut comprendre que Tyler, The Creator (TTC) porte très bien son pseudonyme : Tyler est un créateur. Il écrit toutes les paroles de ses chansons, mais il en signe également la production musicale. Il réalise tous ses vidéoclips ainsi que certains clips de OFWGKTA. Il conçoit ses propres vêtements et est en charge des marques de prêt-à-porter Golfwang et Golf le Fleur. Il crée un festival de musique en 2012, le Camp Flog Gnaw Carnival, et produit l'émission de télévision Loiter Squad (Adult Swim, 2012-2014) avec plusieurs membres de OFWGKTA. TTC est partout, dans tous les médias et surtout toutes les plateformes. Il contrôle entièrement son image. 
Tout comme Vince Staples, TTC se positionne contre une certaine tradition de la représentation dans la culture rap :

D'un côté, le fait d'éviter consciemment la personnalité «thug" ou "ghettocentrique » de rappeurs tels que Snoop Dogg, Jay $\mathrm{Z}$ et plus récemment Waka Flocka Flame peut suggérer un refus, voire une désaffectation envers un nihilisme hyperbolique prévisible et « déjà vu » de ce qui est maintenant une forme culturelle très populaire ${ }^{19}$. (Eate $2013: 545$ )

TTC dépasse le gangsta rap et en redéfinit les codes :

Tyler mobilise des thèmes violents comme une forme d'horreur à l'état pur. Il compense ainsi son sentiment d'échec personnel tout en défiant l'industrie du divertissement, qui exploite régulièrement ces thèmes de façon inconséquente. La façon dont il parle librement de ses émotions et de ses frustrations sert de contrepoint à l'imagerie monstrueuse de sa musique [...]. Le travail de Tyler, The Creator est post-gangsta rap ${ }^{20}$. (Marques $2015: 8$ )

Si tous les rappeurs incarnent un personnage, TTC explore la fragmentation de l'identité du rappeur et décide dès ses premiers albums d'en incarner plusieurs. Il contribue ainsi à l'éclatement de la figure traditionnelle du rappeur. Les explorations de TTC se développent avec la trilogie Wolf, composée de Bastard (2009), Goblin (2011) et Wolf (2013), dans laquelle se développe une narration complexe comprenant une série de personnages : Sam, Tron Cat, Wolf, Dr. TC, Ace, etc. Les propos tenus par TTC et ses personnages sont fortement marqués par de l'ironie, de l'agressivité et du nihilisme. Il devient rapidement ardu de déterminer si et quand TTC est sérieux. Certains de ses personnages ont des rôles qui dépassent ceux de la narration et prennent place dans différents aspects de la carrière de TTC. Par exemple, Ace est le pseudonyme sous lequel il signe la musique de ses albums et il utilise Wolf Haley pour la réalisation de ses clips. L'histoire alambiquée et violente de la trilogie atteint son apogée dans la dernière chanson de Goblin, "Golden", où on apprend que Dr. TC, le thérapeute que Tyler consulte, est en fait Tyler lui-même: "You've been helping yourself this whole time / Your friends? They're just figments of your imagination Dr. TC... See Tyler, I'm your conscience / I'm Tron Cat, I'm Ace, I'm Wolf Haley, I'm... (Me)» (XL Recordings, 2011). La fragmentation de TTC relève d'un tour de force : « Le fait que Dr. TC se révèle être Tyler, The Creator montre également que cette fragmentation est un artifice et qu'elle donne finalement du pouvoir: il n'a pas besoin d'un intermédiaire pour décider/arbitrer à sa place - il est, après tout, maître de la narration ${ }^{21}$ " (Eate 2013 : 535). Même sur le plan fictionnel, Tyler reste le créateur. C'est ce qu'il démontre dans le clip qu'il réalise pour la chanson «Sam (Is Dead) » (The OF Tape vol. 2, Odd Future, 2012). Sam est l'un des personnages que TTC incarne dans la trilogie Wolf. Dans le vidéoclip, on peut voir TTC/Sam qui se fait assassiner par un personnage ressemblant à un dictateur. TTC/Sam est allongé dans la terre puis est rejoint par deux autres personnages incarnés par TTC. L'image révèle ensuite que le dictateur est également incarné par le rappeur. Ce clip sert de démonstration de l'exercice du contrôle de TTC sur ses personnages. À l'inverse d'Ice Cube, TTC utilise sa présence filmique pour fragmenter son personnage musical et non pour en consolider une seule incarnation. La maîtrise qu'il exerce sur son image nous assure que ce constant flou sur son identité est toujours calculé.

$\mathrm{Au}$ fil de sa carrière, TTC délaisse certains personnages et se rend plus vulnérable dans ses chansons. Dans l'album Flower Boy (Columbia, 2017), il est autocritique : "Oh my God, that boy there is so fuckin' lonely / Writin' songs about these people/Who do not exist, he’s such a fuckin' phony » («911/Mr. Lonely », Flower Boy, Columbia, 2017). TTC 
diminue grandement la violence de ses propos, ce qui le rend plus sympathique aux yeux du public et de la critique : "Avant tout, Flower Boy dépeint Tyler comme étant moins tourmenté et tordu [...]. Il laisse aller ses commentaires "no homo" et les alias de Wolf Haley et d'Ace pour révéler ce qu'est peut-être le vrai Tyler. À moins qu'il ne plaisante, ce qui serait l'arnaque de l'année ${ }^{22}$ »(Lester 2017). Malgré ses élans de vulnérabilité, TTC a la réputation d'être imprévisible. C'est pourquoi il est difficile de lui attribuer le même type d'authenticité qui était accordée à Ice Cube : «Le problème d'être un farceur et un provocateur, accidentellement ou non, est que tout le monde pense que vous êtes toujours en train de faire des blagues. Quand les gens se sentent bernés, ils deviennent méfiants. Personne ne croit au garçon qui crie au Loup $^{23} »$ (Jenkins 2017). Qu'il le veuille ou non, TTC reste insaisissable.

21 Avec son album IGOR (Columbia, 2019), TTC s'attribue une nouvelle image et un nouveau personnage. Igor devient le monstre autoréflexif de TTC : le rappeur réinvestit la figure d'Igor, un personnage type de sous-fifre d'histoires d'horreur pour faire écho aux critiques qui ont été émises au début de sa carrière contre son style empruntant au gore. Il apparaît même avec un œil plus gros que l'autre sur la couverture de l'album, rendant la référence plus explicite. Dans Igor, TTC est rejeté d'un triangle amoureux, son orgueil et ses sentiments sont heurtés ; il se sent comme auxiliaire à sa propre vie. Ainsi entre en scène Igor, qui incarne les émotions vécues par TTC : Igor hurle, pleure et se lamente. Il représente pour TTC une figure de dédouanement : c'est lui, et non TTC, qui souffre et fait souffrir les autres. Cette image est particulièrement apparente dans le vidéoclip de la chanson "Earfquake », qui met en scène Igor alors qu'il met le feu au plateau de tournage où il performe. Alors qu'Igor s'évanouit à cause de la fumée, un personnage de pompier, lui aussi incarné par TTC, continue de rapper les paroles de la chanson. Tandis qu'Igor est impulsif et émotif, TTC est là pour reprendre le contrôle de la situation, se plaçant ainsi toujours au centre de la narration.

TTC apparaît comme un perfectionniste qui s'engage dans tous les aspects de la création. Paradoxalement, c'est cette maitrise extrême de son image qui permet de la fragmenter aussi efficacement: il incarne tous les personnages et tous les points de vue. Seul TTC détient tous les morceaux du casse-tête qu'est sa performance; sa démarche autoréférentielle et autocritique agissant comme preuve de l'intelligence artistique qui régit la construction de son univers fictionnel. La multiplication de TTC détourne les bases de l'authenticité des rappeurs et propose un autre modèle de personnage musical: pour mieux s'exprimer, TTC choisit d'en performer plusieurs. Personne ne sait qui est réellement TTC, il se dévoile aussi rapidement qu'il se dérobe à nous. En choisissant d'incarner la multitude, il finit par représenter ses différentes facettes et se sert intelligemment de l'espace audiovisuel pour illustrer ses fragmentations.

\section{Conclusion}

C'est l'espace audiovisuel (film, vidéoclip, présence en ligne) qui consolide les personnages des rappeurs, lesquels y exercent un contrôle sur leur représentation. Dans le cas d'Ice Cube, la crédibilité accordée à la performance constante de son personnage musical consacre l'authenticité comme une valeur capitale de la culture rap : au long de sa carrière et par différents médias, Ice Cube represent. Vince Staples remet en question l'héritage d'Ice Cube en dépassant son ton journalistique. Staples, 
avec des clips à l'esthétique ultra-réaliste, vient s'affirmer comme une présence engagée dans sa communauté, recentrant la valeur d'authenticité véhiculée par le gangsta rap. Staples amincit la ligne entre lui et son personnage musical. À l'inverse, l'espace audiovisuel de TTC explicite la fragmentation de son personnage musical. TTC ne représente rien sinon lui-même, via des personnages multipliés. Son identité complexe présente plusieurs points d'ancrage pour son public, qui peut s'identifier à un personnage plutôt qu'à un autre.

La notion de personnage musical d'Auslander est appliquée ici à la performance de rappeurs, mais s'inscrit dans un héritage de la performance musicale plus global. L'auteur indique qu'il existe un " continuum entre les types de performance musicale dans lesquels les personnages des musiciens sont fortement requis parce qu'ils sont intégrés dans les conventions d'un genre particulier ${ }^{24} »$ (2006: 114). Pour Auslander, " chaque personnage musical existe en relation avec un genre musical, une culture et un public différents ${ }^{25} »(2006: 116)$. Le rappeur détermine son image grâce à sa musique et sa présence scénique, mais également par tout le matériel médiatique qui entoure sa pratique. La performance du personnage musical devient donc continuelle; elle traverse les médias. Il serait approprié de comparer les conditions permettant l'exploration du personnage musical d'un artiste en fonction du genre musical et des médias employés. Auslander porte d'ailleurs une attention singulière à la performance au sein de la musique rock (tout particulièrement, le glam rock : Performing Glam Rock: Gender and Theatricality in Popular Music, 2006), qui, tout comme le rap, donne lieu à des croisements médiatiques permettant aux artistes d'expérimenter avec leur personnage musical. Dans certains cas, ces dynamiques permettent aussi la création de réels personnages, en plus des personnages musicaux: une autre couche de narration s'ajoute à la performance des artistes. C'est sans aucun doute grâce à la créativité d'artistes tels que David Bowie, avec ses personnages Ziggy Stardust et Aladdin Sane, que la musique rap donnera naissance, des décennies plus tard, au Makaveli de Tupac Shakur, au Slim Shady de Eminem et aux Wolf, Ace et Dr. TC de Tyler, the Creator.

\section{BIBLIOGRAPHIE}

Aguirre, Abby, 2016, « Vince Staples is the Anti-Rapper for Our Time », Vogue, 9 septembre 2016, [En ligne], https://www.vogue.com/article/vince-staples-prima-donna-summertime-alexanderwang, consulté le 15 juillet 2021.

Auslander, Philip, 2006, « Musical Personae », TDR, vol. 50, no 1 (printemps), p. 100-119.

Auslander, Philip, 2006, Performing Glam Rock: Gender and Theatricality in Popular Music, Ann Arbor, University of Michigan Press, 2006.

Bell, Christopher J., 2016, « Hip Hop Fancy: Performative Black Masculinity, Feminism, and the Clear Text of Ice Cube's Death Certificate », Mémoire de maîtrise, San Diego, San Diego State University. 
Carissimo, Justin, 2016, « Vince Staples defends Christian Mother Upset Over his Lyrics », The Independant, 5 octobre 2016, [En ligne], https://www.independent.co.uk/arts-entertainment/ music/vince-staples-defends-christian-mother-upset-over-his-lyrics-a7347351.html, consulté le 15 juillet 2021.

Chang, Jeff, 2005, Can't Stop, Won't Stop : A history of the Hip-Hop Generation, New York, St. Martin's Press.

Eate, Penelope, 2013, « Scribblin' Sinnin' Sh*t: Narratives of Rape as Masculine Therapeutic Performance in the Strange Case For and Against Tyler, The Creator ", Journal of African American Studies, vol. 17, $\mathrm{n}^{\circ}$ 4, p. 529-545.

Jenkins, Craig, 2014, « The Personal Reckoning of Tyler, The Creator's Flower Boy », Volture, 28 juillet 2017, [En ligne], https://www.vulture.com/2017/07/review-tyler-the-creator-scum-fck-flower-boy.html, consulté le 15 juillet 2021.

Labalzo Wright, Julie, 2016, « From Boy N the Hood to Hollywood Mogul: Ice Cube's Lasting Stardom in Contemporary Hollywood », dans J. Labalzo Wright et L. Bolton (dir.), Lasting Screens Star: Images That Fade and Persona That Endure, Basingstoke, Palgrave Macmillan, p. 11-26.

Lester, Paul, 2017, « Tyler, the Creator: Flower Boy review - closet door opens to gawky gorgeousness », The Guardian, 21 juillet 2017 [En ligne], https://www.theguardian.com/music/ 2017/jul/21/tyler-the-creator-flower-boy-review, consulté le 15 juillet 2021.

Leonard, David J., 2007, « Ice Cube », dans M. Hess (dir.), Icons of Hip Hop, an Encyclopedia of the Mouvement, Music, and Culture Volume 2, Wesport, Greenwood Press, p. 295-315.

Lessard, Gabriel, 2017, « Du gangsta rap au hip-hop conscient : subversions et alternatives critiques en réponses aux mythes américains », Cahiers d'histoire, vol. 34, no 1, p. 197-217.

Marques, G. S., 2015, « Beyond Gangsta: Hip-Hop, Skate Culture and Web Culture in the Music of Tyler, The Creator ", Art and Design Review, no 3, p. 8-12.

Orejuela, Fernando, 2015, Rap and Hip Hop Culture, New York, Oxford University Press.

White, Simone, 2016, « Vince Staples », Bomb, nº 136, p. 153-159.

\section{Corpus}

Boyz N the Hood, 1991, réal. John Singleton, Columbia Pictures.

Earfquake, 2019, vidéoclip pour Tyler, The Creator, réal. Tyler, The Creator, Columbia, [En ligne], https://youtu.be/HmAsUQEFYGI, consulté le 15 juillet 2021.

Friday, 1995, réal. F. Gary Gray, New Line Cinema.

FUN!, 2018, vidéoclip pour Vince Staples, réal. Calmactic, Def Jam, [En ligne], https://youtu.be/ vz9-pXuvFEU, consulté le 15 juillet 2021.

It Was a Good Day, 1993, vidéoclip pour Ice Cube, réal. F. Gary Gray, Priority Records, [En ligne], https://youtu.be/h4UqMyldS7Q, consulté le 15 juillet 2021.

Norf Norf, 2015, vidéoclip pour Vince Staples, réal. Spike Jordan et Vince Staples, BMI, [En ligne], https://youtu.be/mb6Jc4juSF8, consulté le 15 juillet 2021.

Sam (Is Dead), 2012, vidéoclip pour Domo Genesis et Tyler, The Creator, réal. Tyler, The Creator (Wolf Haley), Odd Future, [En ligne], https://youtu.be/i7qaLXEnrUE, consulté le 15 juillet 2021. 
Tamale, 2013, vidéoclip pour Tyler, The Creator, réal. Tyler, The Creator (Wolf Haley), Odd Future, [En ligne], https://youtu.be/OxlJLz9M8hQ, consulté le 15 juillet 2021.

\section{NOTES}

1. « [They] outmanoeuvred the hip-hop progressives, positioning themselves between the mainstream and those [unheard] voices. No one else, they claimed, was speaking for the brother on the corner but them, loudly, defiantly and unapologetically. So Straight Outta Compton also marked the beginning of hip-hop's obsession with "The Real". From now on, rappers had to represent » (Chang 2005 : 328). Nous traduisons vers le français toutes les citations issues d'ouvrages rédigés en anglais.

2. « the music was undoubtedly difficult to defend " (Chang $2005: 327$ ).

3. « Rap artists such as $2 \mathrm{Pac}$, Bushwick Bill and Ice Cube claim that, in their songs, they are sometimes observers of the environment and sometimes participants. The rapper shows the most graphic images of the street as if he or she is experiencing them firsthand. Consider the fact that all rappers have MC names - alter egos that are much like the characters of professional wrestlers or superhero comics: [...] O'Shea Jackson is not Ice Cube » (Orejuela $2015: 142$ ).

4. « [P]ersonal identity may be seen as something one performs, for instance. One can speak of performing a self in daily life just as readily as one speaks of performing a text in a theatre or concert hall. In short, the direct object of the verb to perform need not be something - it can also be someone, an identity rather than a text » (Auslander 2006 : 101).

5. "when we see a musician perform, we are not simply seeing the "real person" playing; as with actors, there is an entity that mediates between musicians and the act of performance. [...] What musicians perform first and foremost is not music, but their own identities as musicians, their musical personae » (Auslander $2006: 102$ ).

6. "Beginning with the use of the personal pronoun "I," Cube uses a much different tactic to cater to the listener than do the verses of Ren and Eazy: "Fuck the police comin straight from the underground/A young $\mathrm{n}^{* * * *}$ got it bad 'cause I'm Brown" " (Bell 2016 : 21).

7. « The rapper does not end there with his move to make the angst felt by him and his peers understandable to all. By now the listener has bought in to Cube's rhetoric and believes that the rapper is not only rapping about himself, but that he raps for his audience » (Bell $2016: 22$ ).

8. «Because the sound was so powerful that it had to be named, someone called N.W.A.'s music "gangsta rap" after Cube's indomitable anthem » (Chang 2005 : 320).

9. «The success of N.W.A. with Straight Outta Compton reflected the dialectics of their marketing strategy, one that emphasized the truthfulness and authenticity of their music [...]. In other words, the power of Cube's lyrics and of course Cube himself with his perpetual scowl and endless swearing reflected their realness, a fact emphasized by N.W.A. and embraced by listeners » (Leonard $2007: 300$ ).

10. " Doughboy is the embodiment of what Ice Cube had been rapping about: the young black male engaged in gangs and criminal activity, the poverty and desperation of 
being socially marginalised in the hood, the absent father, lack of educational opportunities, and the propensity to end up in jail » (Lobalzo Wright $2016: 58$ ).

11. "Cube's contribution to Boyz transcended his bringing Doughboy to life: he wrote and recorded the film's soundtrack; he gave legitimacy to the film's message and tone given his South Central roots; and his popularity and connection to hip-hop contributed to the media buzz and the film's immense box office success » (Leonard 2007 : 310).

12. «Perhaps to highlight this shift from the typical Ice Cube image, his character Craig wears the same clothes in Friday's opening scene as Doughboy wore in his final scene in Boyz $N$ the Hood. [...] Cube uses Friday to build on the narrative offered in "It Was a Good Day", chronicling the goodness in chillin' with one's homies even on days marked by the most heartache and injustice » (Leonard 2007 : 311).

13. «Although a musician's persona is expected to be more or less continuous from performance to performance, it is also produced at any given performance through the negotiation of a working consensus with the audience. The audience is thus the cocreator of the persona and has an investment in it that extends beyond mere consumption » (Auslander $2006: 117$ ).

14. «I try my best to keep them the same. There are certain things I don't like to share. I don't showcase my family or my personal life, but as far as my music goes, I try to keep it as regular as possible. I'm not rich or famous or important, so I don't try to pretend that I am. [...] That's the problem with rappers, we all have to be rich or famous or tough or some weird shit » (White $2016: 156$ ).

15. Traduction libre: «Un mot de travers, et c'est la débandade / Mets ça sur mon chapeau de Yankee, je suis un gangsta Crip, j'emmerde le gangsta rap. »

16. Traduction libre: "Savoir que le changement va arriver comme Obama et les autres le disent / Mais ils tirent tous les jours autour de ma mère ».

17. "It's not right to attack someone over their stance, their opinions, and their religion. I think that's very immature » (Carissimo 2016).

18. «We already have a lot of issues between black and white relations in this country based on misunderstandings. In my eyes, she doesn't look like a racist. She doesn't look like a mean person. But it's not very responsible for people to try to take that and jump, looking for some sort of commentary on these issues [in the community] (Carissimo 2016).

19. «On the one hand, knowingly and self-consciously eschewing the "thug" persona of more "ghetto-centric" rap performers such as Snoop Dogg, Jay Z and most recently Waka Flocka Flame might suggest a negation of, indeed disaffection with, the predictable "played out" hyperbolic nihilism of what is now a wholly mainstreamed (and mainstreaming) cultural form » (Eate 2013 : 545).

20. "Tyler uses violent themes as pure horror, compensating for his feelings of personal failure and as a challenge to entertainment industry which frequently uses such themes with no greater consequences. The way he speaks freely about his feelings and frustrations serves as a counterpoint to the monstrous imagery that he creates in his music [...]. Tyler, The Creator's work is post-gangsta rap » (Marques $2015: 8$ ).

21. «The fact that Dr. TC is revealed to be Tyler, The Creator also gestures toward this fragmentation as artifice and ultimately empowering: he does not need an interlocutor 
to dictate/arbitrate on his behalf-he is, after all, in control of the narrative » (Eate $2013: 535)$.

22. «But if anything, Flower Boy captures Tyler at his least tormented and twisted [...]. That he's ditched the "no homo" asides and aliases such as Wolf Haley and Ace to reveal the real Tyler underneath, perhaps, is. Unless he's kidding, in which case, it's the con the coup - of the year» (Lester 2017).

23. "The curse of being a jokester and a provocateur, accidentally or on purpose, is that it is assumed that you're "on" all the time. When people feel like they're being worked, they get suspicious. No one believes a boy who cries Wolf » (Jenkins 2017).

24. "There is a continuum from types of musical performance in which the musicians' personae are strongly mandated because they are built into the framing conventions of a particular genre » (Auslander $2006: 114$ ).

25. «For one thing, each persona exists in relation to a different musical genre, culture, and audience » (Auslander $2006: 116$ ).

\section{RÉSUMÉS}

Les rappeurs, en plus d'être des musiciens, sont surtout interprètes. Au long de leur carrière, les rappeurs incarnent sensiblement le même personnage via une diversité médiatique: performance musicale, performance scénique, entrevues, photos, vidéoclips, paroles, etc. Leur rapport au type de personnage change en fonction des modes et des mouvements qui traversent la culture hip-hop : après les personnalités assumées et imposantes des artistes du gangsta rap, la nouvelle génération de rappeurs questionne le personnage habituel du rappeur en en brouillant les frontières et les limites. En plus des paroles, c'est surtout l'espace audiovisuel qui donne aux rappeurs l'occasion de déployer entièrement la mise en scène de leur personnage grâce à l'amalgame des signifiants que les artistes peuvent travailler en combinant texte, image et son. Pour réfléchir à ces questions, cet article propose d'analyser les performances de trois rappeurs de la côte ouest américaine: Ice Cube/O'Shea Jackson (le personnage classique), Tyler, The Creator/Tyler Okonma (le personnage multiple) et Vince/Vincent Staples (le personnage invisible). Il s'agit de démontrer comment O'Shea Jackson continue de performer Ice Cube à travers ses personnages cinématographiques ; comment Vince Staples, en se positionnant contre la tradition du gangsta rap, refuse la notion de personnage ; et comment la multiplicité des rôles créatifs tenus par Tyler, The Creator engendre une multiplicité de personnages.

Rappers, in addition to being musicians, are mostly performers. Throughout their career, rappers play the same character through a diversity of media: musical performance, stage performance, interviews, photos, music videos, lyrics, etc. The relationship to their type of character changes according to the fashions and movements that cross hip-hop culture: after the assumed and imposing personalities of gangsta rap artists, the new generation of rappers question the rapper's usual character by blurring its boundaries and limits. In addition to the lyrics, it is mainly the audiovisual space that gives rappers the opportunity to fully deploy the staging of their character through the combination of texts, images and sounds.

To reflect on these questions, this article proposes to analyze the performances of three West 
Coast rappers: Ice Cube/O'Shea Jackson (the classic character), Tyler, The Creator/Tyler Okonma (the multi-character) and Vince/Vincent Staples (the invisible character). The point is to demonstrate how O'Shea Jackson continues to perform Ice Cube through his cinematographic characters; how Vince Staples, by positioning himself against the gangsta rap tradition, refuses the notion of character; and how the multiplicity of creative roles played by Tyler, The Creator generate a multiplicity of characters.

\section{INDEX}

Keywords : rap, hip-hop, rapper, musical persona, performance, audiovisual space, authenticity Mots-clés : hip-hop, rap, rappeur, personnage musical, performance, espace audiovisuel, authenticité

\section{AUTEUR}

\section{CHARLOTTE GAGNÉ-DUMAIS}

Université de Montréal, Centre de recherche interuniversitaire sur la littérature et la culture québécoise 